\title{
ARTICLE
}

\section{Technical Feasibility of the Dose-to-Curie Conversion Method for a Radwaste Drum Assay}

\author{
Young-Yong JI", Dae-Seok HONG, Il-Sik KANG, and Tae-Kuk KIM \\ PIE \& Radwaste Center, Korea Atomic Energy Research Institute \\ 1045 Daedeokdaero, Yuseong, Daejon, 305-353, Republic of Korea
}

\begin{abstract}
The dose-to-curie (DTC) conversion method is a very simple and easy way to estimate the radioisotope inventory of a drum by a non-destructive assay method. So, in order to make the dose-to-curie conversion method practical, the parameters affecting the measured dose rate were evaluated by using the MCNP code. The major uncertainties associated with measuring the dose rate from a drum could be divided into the impact of the elemental composition of the contained material, and of the spatial distribution of the radioactive sources in a drum. Also, the relative abundance of gamma emitters in a drum, another source of uncertainty at the DTC conversion, could be directly obtained from the measured pulse height spectrum. The dose rate of that drum could then be calculated by using a dose conversion factor which is widely used in the field of environmental radiation measurement. As a result, the impact of the elemental composition and the spatial distribution of radioisotopes within a 200 - $\mathrm{L}$ drum with a low density could be reduced if the detection points are selected at three times the radius of a drum.
\end{abstract}

\section{KEY WORDS: dose-to-cure method, dose rate, MCNP, model drum, dose conversion factor}

\section{Introduction}

The efficient management of radwaste drums is needed to carry out a radionuclide assessment of them, and the results are practically used to confirm the satisfaction of the waste acceptance criteria of the final repository. There are several methods for the non-destructive assay of a radwaste drum that are based on gamma ray scanning and an in-situ object counting system. Although these methods can be processed using a high resolution, a shielded detector, and specific correction techniques, time and cost constraints compared with their accuracy dictate use of a simpler method for practical applications.

The dose-to-curie (DTC) conversion method can simply and easily provide a reasonable estimate of drum activity. It estimates the radioisotope inventory in a radwaste drum using the results of the measured dose rate from it $[1,2]$. This method is based on the fact that the measured dose rate is proportional to the activity in a drum for a given set of parameters, which are the counting geometry, the composition and spatial distribution of the radioisotopes and the waste, and so on. However, it is impossible to meet the same conditions at the field measurement from the radwaste drum, especially the radioisotope distribution. Therefore, it is necessary to evaluate the assumption and the limitations on the dose rate measurement for a DTC conversion.

In this study, the accuracy and limitations associated with the parameters affecting the results were evaluated by using the MCNP code. The location of the detector from the drum surface and the measuring method of the dose rate were determined to minimize the effects on the inhomogeneous distribution of the radioisotopes in a drum used in KAERI.

\section{Dose-to-Curie Conversion Method}

\section{Mathematical expression}

The measured dose rate is generally proportional to the activity for gamma emitters in a drum at given parameters. It is therefore possible to estimate the curie inventory due to gamma emitters in a drum based on the measured dose rate from it. And also, if the relative abundance of gamma emitters is known from another method, the quantitative analysis of each gamma emitter could be carried out from the curie inventory and the relative abundance.

The dose-to-curie conversion method conducts the above procedure to assay the radwaste drum. Its mathematical expression is as follows:

$$
A=\frac{D}{\sum d_{i} f_{i}}
$$

where, $\mathrm{A}$ is the total activity $(\mathrm{mCi})$ of a drum due to gamma emitters, $D$ is the dose rate $(\mathrm{mR} / \mathrm{h})$ measured from a drum, $f_{i}$ is the relative abundance of the gamma emitter existing in a drum, and $d_{i}$ is the dose rate per unit curie $(\mathrm{mR} / \mathrm{h} / \mathrm{mCi})$ for each gamma emitter and is calculated from a theoretical analysis known as the Monte Carlo simulation.

Once the total activity, $\mathrm{A}$, is obtained, the individual activity, $A_{i}$, for each gamma emitter in a drum is calculated by using the relative abundance of the gamma emitter, as shown in equation (2).

$$
A_{i}=A f_{i}
$$

*Corresponding author, Tel. +82-42-868-4958, Fax. +82-42-8611790, E-mail: yyji@kaeri.re.kr 


\section{Parameters affecting dose rate measurement}

The DTC method is simple and easy to make a reasonable estimate of the drum activity when properly applied. To make the DTC method practical, numerous assumptions had to be made and limitations existed on its use. These assumptions and limitations are related to the dose rate measurement. Therefore, it is necessary to understand and evaluate the parameters affecting the measured dose rate.

The sources of uncertainty associated with measuring the dose rate from a drum can be divided into two categories: the impact of the elemental composition and of the spatial distribution of radioisotopes in a drum. In the case of the elemental composition in a drum, the measured dose rate could be differently depending on the potential existence of voids, the density and the volume of the contents of a drum. The result of dose rate from the field measurement could also be affected by the distribution of radioisotopes within a drum.

The basic assumption for the DTC method is that the elemental composition and the radioisotopes are uniformly distributed throughout a drum. Therefore, these assumptions have to be investigated as to whether it is applicable to the field measurement for the real drum of which the hot spot of the element or the radioisotopes could exist.

\section{Dose rate per unit curie}

The DTC conversion method demands in advance the data regarding the dose rate per unit curie for possible radioisotopes in a drum to be assayed. This data could be calculated from the Monte Carlo simulation. Therefore, the dose rate per unit curie was calculated by the MCNP code for the combustible DAW (dry active waste) drum with a density of about $0.25 \mathrm{~g} / \mathrm{cm}^{3}$ and for the soil drum with a density of about $1.35 \mathrm{~g} / \mathrm{cm}^{3}$. The specification of each drum for the MCNP modeling is shown in Table 1.

Table 1 Specification of the drum for MCNP modeling

\begin{tabular}{|c|c|c|c|}
\hline \multicolumn{2}{|c|}{ Drum } & DAW & SOIL \\
\hline \multicolumn{2}{|c|}{ Elemental composition } & $\mathrm{CH}_{2}$ & $\mathrm{SiO}_{2}$ \\
\hline \multicolumn{2}{|c|}{ Density } & $0.25 \mathrm{~g} / \mathrm{cm}^{3}$ & $1.35 \mathrm{~g} / \mathrm{cm}^{3}$ \\
\hline \multirow{3}{*}{ Dimension } & Radius & \multicolumn{2}{|c|}{$28.72 \mathrm{~cm}$} \\
\cline { 2 - 4 } & Height & \multicolumn{2}{|c|}{$87.30 \mathrm{~cm}$} \\
\cline { 2 - 4 } & Thickness & \multicolumn{2}{|c|}{$0.12 \mathrm{~cm}$} \\
\hline
\end{tabular}

The density values of each drum are the average densities from the waste generated at KAERI. It is assumed that all drums have a capacity of 200 L fully filled with contents, and all radioisotopes are uniformly distributed in a drum. Then, four calculation surfaces from the outside of a drum at the tally process of the MCNP were selected at 1, 2, 3, and 4 times the radius from center of a drum, respectively. The simulated radioisotopes were selected at a low energy range as a $\mathrm{Ba}-133$, at a middle range as a $\mathrm{Cs}-137$ and at a high energy range as a Co-60. Table 2 shows the calculation results obtained from the MCNP code.
Table 2 Calculated dose rate per unit curie at several distances from the center of a $200-\mathrm{L}$ drum

\begin{tabular}{|c|c|c|c|c|c|}
\hline & \multirow{2}{*}{$\begin{array}{l}\text { Radio- } \\
\text { isotope }\end{array}$} & \multicolumn{4}{|c|}{ Dose rate per unit curie $(\mathrm{mR} / \mathrm{h} / \mathrm{mCi})$} \\
\hline & & $1 \mathrm{r}^{*}$ & $2 \mathrm{r}$ & $3 \mathrm{r}$ & $4 \mathrm{r}$ \\
\hline \multirow{3}{*}{$\begin{array}{l}\mathrm{D} \\
\mathrm{A} \\
\mathrm{W}\end{array}$} & Ba-133 & $\begin{array}{c}1.2815 \\
(0.14 \%)^{* *}\end{array}$ & $\begin{array}{c}0.3860 \\
(0.15 \%)\end{array}$ & $\begin{array}{c}0.1880 \\
(0.18 \%)\end{array}$ & $\begin{array}{c}0.1101 \\
(0.21 \%)\end{array}$ \\
\hline & Cs-137 & $\begin{array}{c}2.2281 \\
(0.10 \%)\end{array}$ & $\begin{array}{c}0.6576 \\
(0.12 \%)\end{array}$ & $\begin{array}{c}0.3182 \\
(0.15 \%)\end{array}$ & $\begin{array}{c}0.1857 \\
(0.18 \%)\end{array}$ \\
\hline & Co-60 & $\begin{array}{c}9.4220 \\
(0.10 \%) \\
\end{array}$ & $\begin{array}{c}2.7468 \\
(0.12 \%) \\
\end{array}$ & $\begin{array}{c}1.3236 \\
(0.15 \%) \\
\end{array}$ & $\begin{array}{c}0.7745 \\
(0.18 \%)\end{array}$ \\
\hline \multirow{3}{*}{$\begin{array}{l}\mathrm{S} \\
\mathrm{O} \\
\mathrm{I} \\
\mathrm{L}\end{array}$} & Ba-133 & $\begin{array}{c}0.5151 \\
(0.22 \%) \\
\end{array}$ & $\begin{array}{c}0.1588 \\
(0.24 \%) \\
\end{array}$ & $\begin{array}{c}0.0781 \\
(0.27 \%)\end{array}$ & $\begin{array}{c}0.0458 \\
(0.30 \%)\end{array}$ \\
\hline & Cs-137 & $\begin{array}{c}1.0250 \\
(0.18 \%) \\
\end{array}$ & $\begin{array}{c}0.3102 \\
(0.19 \%) \\
\end{array}$ & $\begin{array}{c}0.1517 \\
(0.22 \%) \\
\end{array}$ & $\begin{array}{c}0.0886 \\
(0.26 \%) \\
\end{array}$ \\
\hline & Co-60 & $\begin{array}{c}4.8979 \\
(0.17 \%)\end{array}$ & $\begin{array}{c}1.4621 \\
(0.18 \%)\end{array}$ & $\begin{array}{c}0.7111 \\
(0.22 \%)\end{array}$ & $\begin{array}{c}0.4164 \\
(0.25 \%)\end{array}$ \\
\hline
\end{tabular}

$* \mathrm{r}:$ the radius of a drum, $* *(\mathrm{)})$ : the relative error

\section{Relative abundance of gamma emitters in a drum}

In order to estimate the radioisotope inventory of a drum from the DTC conversion method, the measured dose rate as well as the relative abundance of gamma emitters in a drum is a very important factor to be evaluated because of the direct linearity between the measured dose rate and the gamma emitters in a drum. The dose rate is directly measured with the survey meter. However, the relative abundance of gamma emitters in a drum to be assayed is determined from an indirect measurement using the reference data that is obtained from the analysis results of the sample taken at another representative drum or the data of the material balance by the waste stream. From this reason, the relative abundance in the real drum could be different from the reference data obtained by an indirect measurement.

The uncertainty of the radioisotope inventory of the assayed drum from the DTC conversion method could be increased because of the different detection mechanism between the dose rate and the relative abundance of gamma emitters in a drum. Unfortunately, that expands the limitation of using the DTC method. It is, therefore, necessary to find out a suitable measurement method of which two variables could be obtained from the drum to be assayed at once.

This method could be realized by using the dose conversion factor, $G(E)$, that has been widely used in the field of the environmental radiation measurement[3]. The dose rate from a drum could be directly calculated from the measured gamma ray spectra by using the dose conversion factor of equation (3):

$$
\dot{X}=\frac{1}{T_{c}} \int_{E_{0}}^{E_{1}} N(E) G(E) d E
$$

where, $T_{c}$ is the counting time ( $\left.\mathrm{sec}\right), \mathrm{N}(\mathrm{E})$ is gamma ray counts per unit energy width (counts $/ \mathrm{keV}$ ) in a measured pulse height spectrum, and $\mathrm{G}(\mathrm{E})$ is the dose conversion factor at a specified geometry of gamma ray spectroscopy, which is a function of the detector response. 
The response function matrix for several incident gamma energies at a fixed geometry can be calculated by using the Monte Carlo simulation. From the calculated 50x50 response matrix for a $3 " \varphi \times 3 " \mathrm{NaI}(\mathrm{Tl})$ scintillation detector, the dose conversion factor was calculated as a function of the incident gamma ray energy, as shown in Fig. 1. The mass energy absorption coefficient in dry air, which is one column vector, was used as the conversion coefficient of the gamma ray flux incident on the detector into the exposure dose rate. From this factor, it was possible to obtain the relative abundance of gamma emitters in a drum as well as the dose rate from it at once.

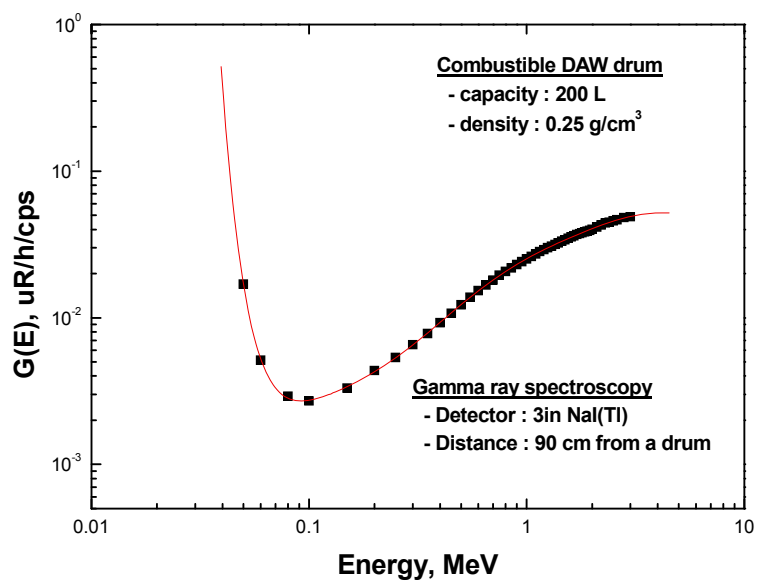

Fig. 1 The dose conversion factor for the measured spectrum of a of a $3 " \varphi \times 3 " \mathrm{NaI}(\mathrm{Tl})$ scintillation detector to exposure dose rate from a-200 L combustible DAW drum

\section{Results and Discussion}

The dose rate resulting from gamma rays emanating from a waste drum is a function of the distance from the drum. The detector location should be selected to help minimize errors caused by variations in actual waste and radioisotope spatial distributions with respect to the assumed distributions. And then, it is necessary to evaluate the major uncertainties associated with measuring the dose rate from a drum.

\section{Impact of off-centered radioisotopes}

In order to identify the variation of the measured dose rate due to the distribution of hot spots in a drum, the offcentered hot spots were individually located by inserting the mixed point source from $5 \mathrm{~cm}$ to $25 \mathrm{~cm}$ horizontally away from the center of a drum, as shown in Fig. 2. After inserting the mixed standard source, which consists of Ba-133, Cs137 , and Co-60, into the off-centered hole, the dose rates were measured at four horizontal points at several distances from a drum. The total activity of a drum was then estimated from the average value of the measured dose rates by using the DTC method.

The results for the DAW drum are shown in Fig. 3. In the case of the detection point on the surface, the difference between the measured and the real value was generally above $20 \%$. On the other hand, the difference gradually decreased as the detection points were far away from the drum. The uncertainty due to the non-uniformly distributed radioisotopes was then greatly reduced where the detection points were at three times the radius of the drum.

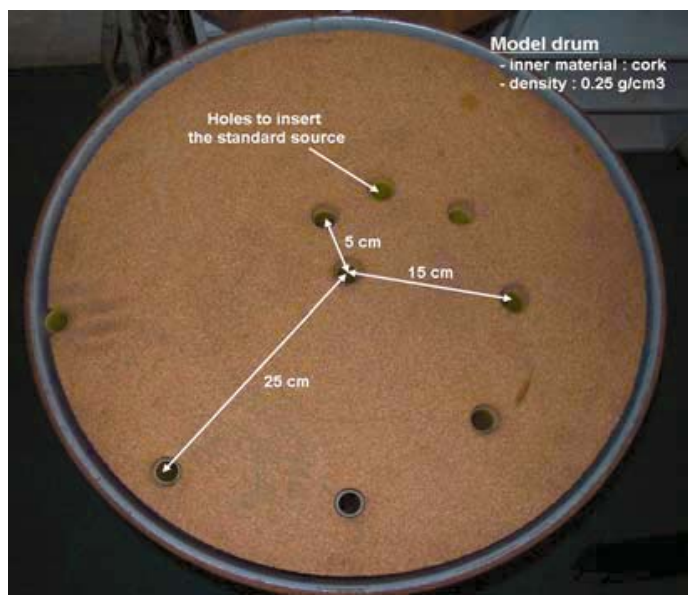

Fig. 2 The model drum that represents the combustible DAW drum with a density of about $0.25 \mathrm{~g} / \mathrm{cm}^{3}$

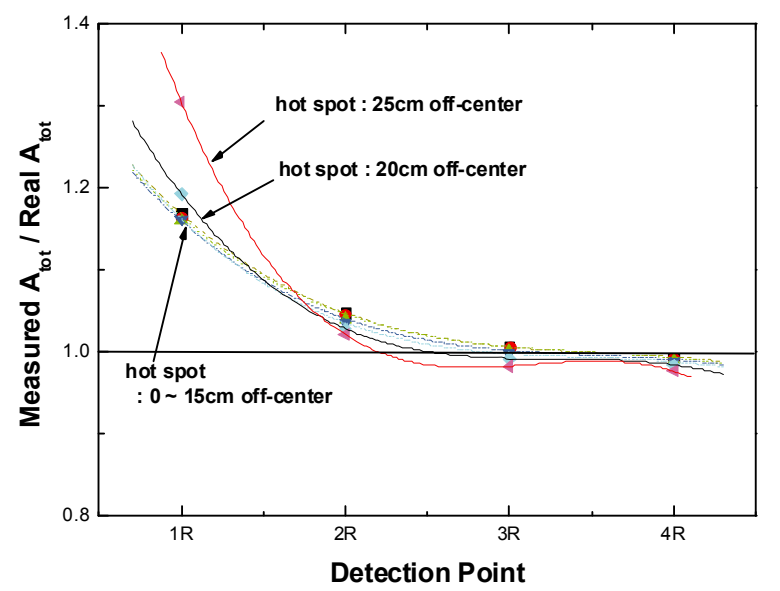

Fig. 3 The difference between the measured and the real total activity of a standard source inserted into a model drum according to the distance away from the center of a drum.

\section{Impact of voids in a drum}

The dose rate per unit curie was calculated from the contents in a drum that was $100 \%$ filled. Although the field measurement for the dose rate should ideally be met with the same condition, void regions within the real waste drum might exist. In order to estimate the uncertainty introduced by the possible existence of void regions, the dose rate from the simulated drums, of which the void regions ranging from $10 \%$ to $30 \%$ for total volume were introduced at three locations within a $200-\mathrm{L}$ drum, was calculated by the MCNP code. The density of the rest of the void regions was increased to maintain the same mass of waste in the drum. The three voids were of a cylindrical type with the same inner diameter of a drum and located at the upper, middle, and lower parts of the drum.

The conducted calculation assumed that the mixed source 
of $1 \mathrm{mCi}$ which consisted of Ba-133, Cs-137, and Co-60 was uniformly distributed in the contents region except for the void. The heights of the cylindrical void regions were the same at the three positions and changed with the void amount. For example, the dimension of one cylindrical void in the case of $10 \%$ void region was about $28.6 \mathrm{~cm}$ in radius and $2.9 \mathrm{~cm}$ in height. Results of the calculated dose rate due to the void regions are shown in Fig. 4. The impact of the void regions seldom appeared in the combustible DAW drum. However, in the case of the soil drum, the difference between without the void and with $30 \%$ of void regions was about $15 \%$.

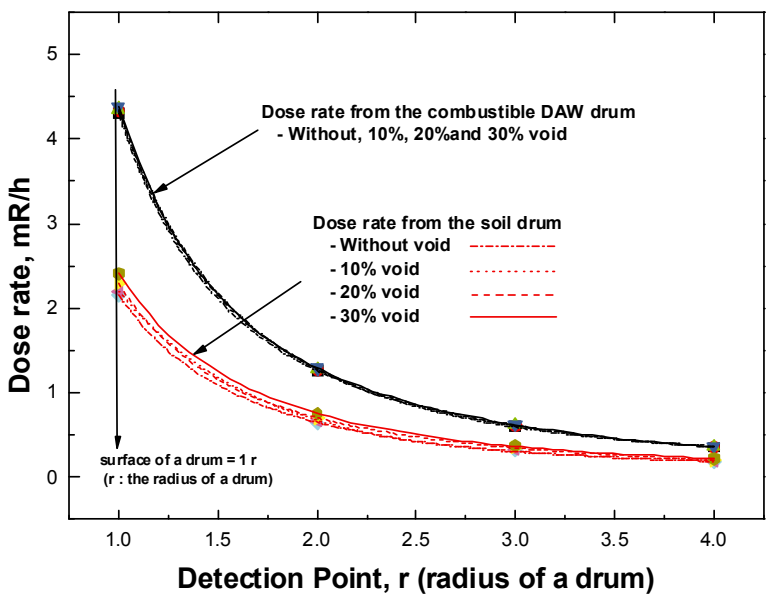

Fig. 4 The impact of voids in a drum (combustible DAW, soil) with the detection point of surface dose rate

\section{Density variation}

Since the measured dose rate is sensitive to the density of a drum, the dose rate per unit curie, which is sort of a calibration factor to convert the measured dose rate into the total activity of a drum, has to meet the same density condition with a drum to be assayed. The combustible DAW drum with a capacity of $200 \mathrm{~L}$ generally has a density of 0.2 to $0.5 \mathrm{~g} / \mathrm{cm}^{3}$, and the soil drum with the same capacity has a density of 1.0 to $1.5 \mathrm{~g} / \mathrm{cm}^{3}$. Therefore, the calibration factors for the measured dose rate according to the variation in the density of the real drum should be prepared in advance in order to make this DTC conversion more practical.

The values of the dose rate per unit curie for drums with several densities were calculated by the same condition as in the former calculation. Those values as a function of the density variation were then obtained through a non-linear curve fitting process. The results assessed at three times the radius from the center of a drum are shown in Fig. 5. From the fitted curve, the calibration factor had to be selected in accordance with the real density of a drum. Those curves were fitted from 0.2 to $1.5 \mathrm{~g} / \mathrm{cm}^{3}$, and all data showed good agreement for the fitting results in spite of the DAW drum having a different atomic number from the soil drum. That meant that the uncertainty due to the variation of the elemental composition in a drum rarely affects the DTC conversion.

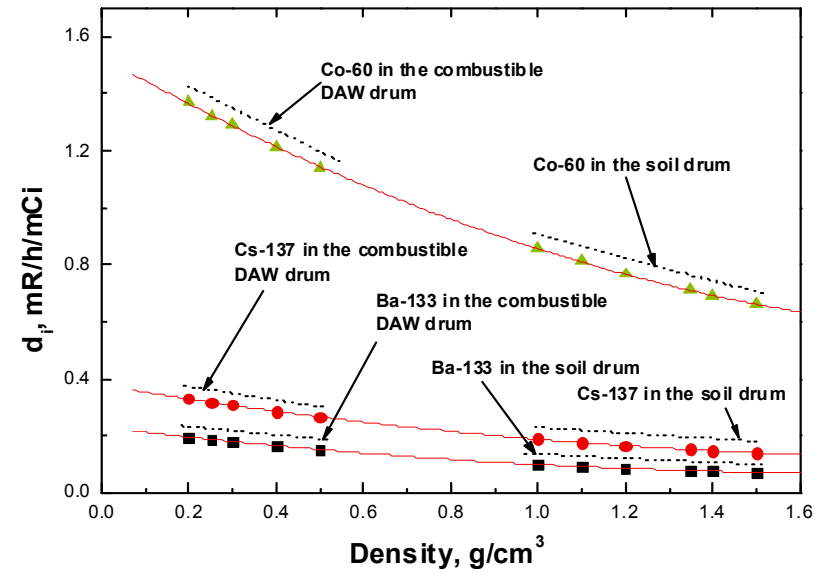

Fig. 5 The dose rate per unit curie as a function of the density variation of a $200-\mathrm{L}$ drum at three times the radius from the drum center

\section{Conclusion}

In order to make the dose-to-curie conversion method practical, the parameters affecting the measured dose rate were evaluated by using the MCNP code. The major uncertainties associated with measuring the dose rate from a drum could be divided into the impact of the elemental composition of the contained material and the spatial distribution of radioisotopes in a drum. As a result, the variation of the calculated dose rate due to the impact of the void region in the contents of a drum seldom appeared in the low density drum. However, in the case of a high density of about $1.35 \mathrm{~g} / \mathrm{cm}^{3}$, the variation of the dose rate due to the voids had to be examined. The dose rate per unit curie, which is a calibration factor, also had to be selected in accordance with the real density of the contents from the fitted equation of the curve with the density variation. The impact on the spatial distribution of radioisotopes within a 200-L drum with a low density could be reduced where the detection points were selected at three times the radius of a drum. The relative abundance of gamma emitters in a drum, which was another source of uncertainty of the DTC conversion, was directly obtained at the measured pulse height spectrum. The dose rate of that drum could then be calculated by using the dose conversion factor at once.

\section{Reference}

1) S.R. Gedeon, Basic for Dose Rate to Curie Assay Method, WHC-SD-WM-RPT-267, Rev. 0, Westinghouse, (1996).

2) R.S. Howell, Guidance on Dose Rate Measurements for Use in Dose-to-Curie Convesions, Westinghouse, (2000).

3) J.S. Jun, C.Y. Yi, H.S. Chai and H. Cho, "Calculation of Spectrum to Dose Conversion Factors for a $\mathrm{NaI}(\mathrm{Tl})$ Scintillation Detector Using the Response Matrix," J. Korean Physics Society, 28[6],716 (1995). 